\title{
FRECUENCIA DE HUEVOS DE Toxocara canis EN PARQUES PÚBLICOS DE MEXICALI, BAJA CALIFORNIA, MÉXICO
}

Frequency of Toxocara canis eggs in public parks of Mexicali, Baja California, Mexico

\section{Leticia RAMÍREZ-RUBIO ${ }^{1 *}$, Onofre Rafael GARCÍA-CUETO ${ }^{1}$, Luis TINOCO-GRACIA ${ }^{2}$, Margarito QUINTERO-NÚÑNEZ ${ }^{3}$, Sergio Arturo CUETO-GONZÁLEZ ${ }^{2}$ y Enrique TRASVIÑA-MUÑOZ ${ }^{2}$}

\footnotetext{
${ }^{1}$ Instituto de Ingeniería, Universidad Autónoma de Baja California, Blvd. Benito Juárez y calle de la Normal s/n, Col. Insurgentes Este, 21280 Mexicali, Baja California, México

${ }^{2}$ Instituto de Investigaciones en Ciencias Veterinarias, Universidad Autónoma de Baja California, km. 3.5 carretera San Felipe, Fracc. Campestre, 21387 Mexicali, Baja California, México

${ }^{3}$ Secretaría de Protección al Ambiente de Baja California. Blvd. Benito Juárez 1 esq. con Francisco L. Montejano, Col. Esteban Cantú, 21260 Mexicali, Baja California, México

*Autor para correspondencia: leticia.ramirez.rubio@uabc.edu.mx
}

(Recibido octubre 2017; aceptado septiembre 2018)

Palabras claves: parásitos, helmintiasis, suelo, salud pública, perros

\section{RESUMEN}

Se realizó un estudio en 56 parques públicos de la ciudad de Mexicali, Baja California, México, con la finalidad de conocer el nivel de contaminación del suelo con huevos de Toxocara canis. La selección de parques fue aleatoria y la duración del muestreo para estimar la variabilidad de la contaminación respecto a las estaciones fue de un año. Las muestras de suelo fueron analizadas con la técnica de flotación con solución salina saturada. Se encontró que la frecuencia de parques contaminados fue de $54 \%$ y que la contaminación especifica más frecuente por parásito se debió a Toxocara canis con $46.4 \%$, nivel menor en $16.1 \%$ comparado con un estudio realizado en 2007. Asimismo, se encontraron otros parásitos como Ancylostoma caninum (5.3\%) y finalmente, en un solo parque, Spirocerca spp. y Strongylus spp. (1.78 \%). La concentración de contaminación por huevos de T. canis fue de 1.6 huevos por $66 \mathrm{~g}$ de suelo, valor bajo en comparación con otros estudios en el país y el mundo; aún así, representa un riesgo para la salud pública local.

Key words: parasites, helminthiasis, soil, public health, dogs

\begin{abstract}
A study was carried out in 56 public parks of the city of Mexicali, Baja California, Mexico to know the level of soil contamination by Toxocara canis eggs. The selection of parks was random and the duration of the sampling to estimate the variability of pollution with respect to the seasons was one year. The soil samples were analyzed using the flotation technique with saturated saline solution. It was found that the frequency of contaminated parks was $54 \%$, and specific contamination per parasite was found to be more frequent by Toxocara canis with $46.4 \%$, which was $16.1 \%$ lower compared to a study carried out in 2007. Likewise, other parasite's eggs like Ancylostoma caninum
\end{abstract}


(5.3\%) were found, and one park was parasitized with Spirocerca spp. and Strongylus spp. (1.78\%). The contamination rate for T. canis eggs was 1.6 eggs per $66 \mathrm{~g}$ of soil, i.e., a low level of contamination compared to other studies in the country and in the world, even though it still represents a risk for public health.

\section{INTRODUCCIÓN}

En el mundo aproximadamente 1500 millones de personas, es decir, casi el $24 \%$ de la población mundial, está infectada con parásitos intestinales transmitidos por el suelo (OMS 2017). Las heces de perros son una de las principales fuentes de contaminación del suelo, ya que al ser depositadas pueden llegar a portar parásitos zoonóticos (Kazacos 2000). Este problema ha ido en aumento conforme ha crecido la población canina en situación de calle y la tenencia irresponsable de mascotas, aunada a medidas sanitarias deficientes en el manejo de las heces (Katagiri 2008).

Uno de los parásitos más frecuentes que transmiten los perros y que resulta de interés para la salud pública es Toxocara canis, ya que sus huevos contaminan el medio ambiente. Estos parásitos tienen la capacidad de causar trastornos en diferentes órganos como los ojos, donde pueden albergarse provocando granulomas e incluso ceguera. Otras patologías ocasionadas por este helminto son afecciones de la piel como dermatitis y problemas respiratorios (Holland 2005).

La toxocariasis humana está presente en todo el mundo, y es más probable que los niños desde los pocos meses de edad hasta los 4 a 5 años estén expuestos al riesgo de infección dados sus hábitos de geofagia (Carvalho 2011). En Estados Unidos, al menos setenta niños cada año padecen ceguera por esta parasitosis (CDC 2017). En México sólo existen reportes bibliográficos de casos aislados de infección por larva migrans ocular y larva migrans visceral, que no indican la magnitud del problema. Sin embargo, en estudios serológicos recientes se han reportado prevalencias del $22.22 \%$ en Ecatepec, Estado de México (Romero 2013), de $26.2 \%$ en la población rural Tepehuanos, Durango (Alvarado-Esquivel 2014), y de $12.02 \%$ en Amecameca y Chalco, Estado de México (Nava 2015). Es importante destacar que dichas prevalencias demuestran la exposición de las personas al parásito, más no el desarrollo de la enfermedad. Los huevos de Toxocara canis pueden encontrarse en el suelo de gran parte del planeta y aparecen de 2-88 \% en muestras de tierra recogidas en varios países y regiones. Las altas temperaturas y la humedad ambiental de los trópicos favorecen la transmisión de la especie (ISUCFSPH 2016). La mayoría de los casos de larva migrans en humanos se reporta en el sur y este de Estados Unidos, Europa oriental y occidental, el Caribe, Hawái, Australia, Filipinas y Sudáfrica (GIDEON 2017).

Dado que es una enfermedad poco estudiada, en 2007 se realizó una investigación en 32 parques públicos de la ciudad de Mexicali, donde se registró una frecuencia de contaminación por Toxocara canis en el suelo del $62.5 \%$ (Tinoco 2007), mientras que la seroprevalencia en niños fue del $10.6 \%$ (Tinoco 2008). Esto confirma que la población está expuesta al parásito. Sin embargo, debido al escaso conocimiento de la sintomatología que causa, en dicha ciudad no se tienen cifras oficiales, por lo que podría existir el riesgo de un diagnóstico incorrecto.

Tomando en cuenta que durante 2013 en Baja California las helmintiasis ocuparon el lugar 16 entre los padecimientos crónicos degenerativos (Gobierno del Estado de Baja California 2013), se enfatiza la importancia del presente estudio, cuyo objetivo es estimar el nivel de contaminación por huevos de Toxocara canis en suelo de los parques de Mexicali.

\section{MATERIALES Y MÉTODOS}

\section{Diseño del estudio}

Se realizó un estudio transversal en 56 parques públicos seleccionados aleatoriamente en la ciudad de Mexicali, Baja California, de febrero 2016 a enero 2017, para conocer la influencia estacional sobre el riesgo potencial de infección con huevos de parásitos intestinales de perro en el suelo.

Se obtuvieron 10 muestras de suelo por cada parque, sumando 560 muestras en total. Cada parque fue muestreado sólo una vez. Se eligió a los parques como área de estudio debido a que las heces se quedan más tiempo expuestas en el ambiente, lo que aumenta la probabilidad de contaminar el suelo.

Para determinar la frecuencia de parques contaminados con huevos de Toxocara canis se consideraron las variables tipo de suelo, estación del año y nivel socioeconómico. Para tipo de suelo se consideraron dos categorías: suelo desnudo (tierra sin pasto) y con 
pasto. Además, se incluyeron las cuatro estaciones del año. La ubicación socioeconómica se determinó con un plano de tipología de vivienda del Instituto Municipal de Investigación y Planeación Urbana de Mexicali (IMIP, 2016). Las categorías fueron progresiva, popular, interés social, media y residencial.

\section{Toma de muestras de suelo}

Se obtuvieron 10 muestras de cada dirección cardinal y del centro del parque, sumando 10 muestras por parque. Cada muestra de suelo consistió en la recolección de cuadros de tierra de $20 \times 20 \mathrm{~cm}$, con una profundidad de 1 $\mathrm{cm}$, que representan aproximadamente $400 \mathrm{~g}$. El suelo fue extraído con una cuchara de metal, previamente lavada y desinfectada con hipoclorito sódico al $1 \%$ y guardado en bolsas de plástico con cierre hermético, previamente identificadas (Tinoco 2007). Posteriormente fueron analizadas en el Instituto de Investigaciones en Ciencias Veterinarias de la Universidad Autónoma de Baja California.

\section{Técnicas parasitológicas}

Para su análisis parasitoscópico, las muestras se colocaron en recipientes de $1 \mathrm{~L}$ y se sometieron a la técnica de flotación con solución salina (densidad específica de 1.20). Cada análisis consistió en la homogeneización de $66 \mathrm{~g}$ de suelo diluido en $1 \mathrm{~L}$ de solución salina. Posteriormente se examinaron las muestras con base en las características descritas previamente (Zajac 2012).

Se consideraron como positivos a Toxocara canis todos aquellos parques en los que se observó cuando menos un huevo de este parásito en cualquiera de las 10 muestras de suelo levantadas.

\section{Análisis estadístico}

Los resultados del análisis inferencial entre la frecuencia de parques contaminados por huevos de Toxocara canis y las variables mencionadas fue estimado mediante la prueba de chi cuadrada. El análisis estadístico fue realizado con el programa Statistix 9.0®.

\section{RESULTADOS}

La frecuencia general de parques contaminados en Mexicali, BC, fue de $54 \%$ (30/56). Fueron 66 muestras las que se encontraron contaminadas, lo que representa el $11.78 \%$ (66/560). Con base en la frecuencia de contaminación especifica por parásito se encontró que el más frecuente fue Toxocara canis con $46.4 \%$ (26/56), seguido por Ancylostoma caninum $5.3 \%$ (3/56) y por Spirocerca spp. y Strongylus spp. Estos dos últimos se encontraron en un solo parque (Fig.1)

Se observó una diferencia significativa $(\mathrm{p}<0.01)$ en la relación de muestras contaminadas $(66 / 560)$ con la estación del año, siendo en la primavera cuando hay mayor riesgo de infección (3.7 veces más respecto al invierno) (Cuadro I).

La ubicación socioeconómica no fue significativa en el análisis por muestras contaminadas, aun cuando presentaron un riesgo mayor las colonias de nivel progresivo con el $20 \%$, (Cuadro II).

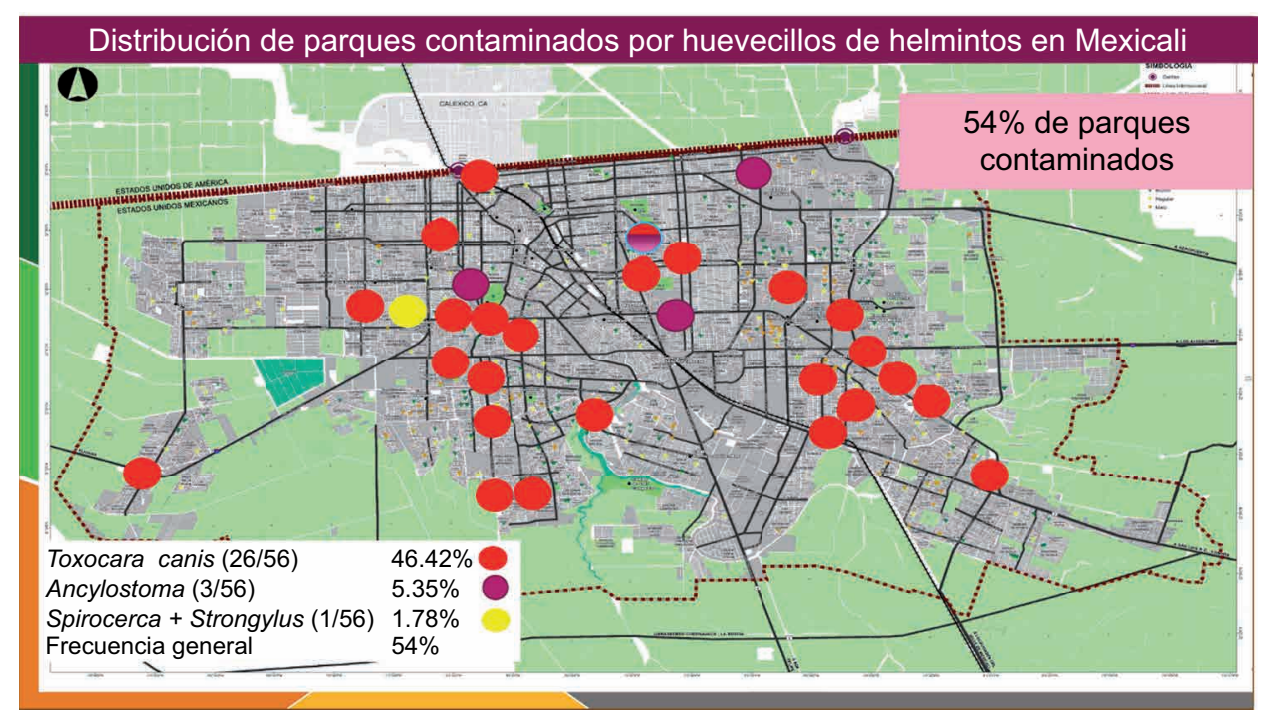

Fig. 1. Mapa de distribución de parques contaminados por huevos de helmintos en Mexicali 
CUADRO I. MAGNITUD DEASOCIACIÓN ENTRE MUESTRAS CONTAMINADAS Y LA ESTACIÓN DEL AÑO

\begin{tabular}{lrrrlcl}
\hline Estación & $(+)$ & $(-)$ & Total & OR & IC 95\% & P \\
\hline Invierno & 4 & 86 & 90 & 1 & Referencia & \\
Otoño & 12 & 118 & 130 & 2.1 & $(0.6-7)$ & 0.17 \\
Verano & 4 & 26 & 30 & 3.3 & $(0.7-14.1)$ & 0.09 \\
Primavera & 46 & 264 & 310 & 3.7 & $(1.3-10.7)$ & $0.01 *$ \\
\hline
\end{tabular}

*Comparación de muestras contaminadas de suelo con base la estación del año. Resultados de la prueba $\chi^{2}$ (p) y razón de oportunidad (OR) utilizando un intervalo de confianza (IC) del 95\%

CUADRO II. MUESTRAS CONTAMINADAS POR ESTATUS ECONÓMICO

\begin{tabular}{lrrrl}
\hline Estatus & $(+)$ & \multicolumn{1}{c}{$(-)$} & Total & $\%$ \\
\hline Progresivo & 2 & 8 & 10 & 20 \\
Popular & 25 & 205 & 230 & 10.86 \\
Interés social & 23 & 177 & 200 & 11.5 \\
Medio & 13 & 87 & 100 & 13 \\
Residencial & 3 & 17 & 20 & 15 \\
\hline
\end{tabular}

Con relación al tipo de suelo, el $8.1 \%$ de las muestras positivas se detectó en suelo desnudo, mientras que el $18.6 \%$ se detectó en suelo con pasto. Resultó 2.5 veces mayor la probabilidad de encontrar parásitos en suelo con pasto que en suelo desnudo (Cuadro III).

CUADRO III. CONTAMINACIÓN POR TIPO DE SUELO

\begin{tabular}{lcccccc}
\hline $\begin{array}{l}\text { Tipo } \\
\text { suelo }\end{array}$ & $(+)$ & $(-)$ & $\begin{array}{l}\text { Total } \\
(\%)\end{array}$ & OR & IC 95\% & P \\
\hline $\begin{array}{l}\text { Suelo } \\
\text { desnudo }\end{array}$ & 30 & 337 & $367(8.1)$ & 1 & Referencia & \\
\hline $\begin{array}{l}\text { Suelo } \\
\text { con pasto }\end{array}$ & 36 & 157 & $193(18.65)$ & 2.5 & $(1.5-4.3)$ & $0.003^{*}$ \\
\hline
\end{tabular}

La intensidad de contaminación del suelo se clasificó de acuerdo con el número de muestras positivas de un total de 10 por parque. Baja (una a tres muestras positivas), mediana (cuatro a seis muestras positivas) y alta (siete a 10 muestras positivas). La densidad media de huevos por muestra en los parques de Mexicali fue de 1.6 huevos/66 g de suelo.

La intensidad de contaminación fue baja en 25 parques $(44.6 \%)$ mientras que tres parques $(5.3 \%)$ resultaron medianamente contaminados y dos (3.5\%) altamente contaminados (Cuadro IV).
CUADRO IV. INTENSIDAD DE CONTAMINACIÓN DEL SUELO POR ESPECIE DE PARÁSITO

\begin{tabular}{lccc}
\hline Intensidad & Toxocara & Ancylostoma & Spirocerca spp. \\
\hline Bajo & 23 & 2 & 0 \\
Media & 2 & 0 & 1 \\
Alta & 1 & 1 & 0 \\
\hline
\end{tabular}

\section{DISCUSIÓN}

A pesar de que los gatos y los perros son los huéspedes habituales de Toxocara, el contacto directo no es considerado un riesgo potencial. Los huevos eliminados en las heces requieren un periodo de 4 a 6 semanas para ser infecciosos, por lo que el suelo de los parques es una fuente potencial de contaminación.

La importancia del suelo contaminado por huevos de T. canis como vehículo capaz de transmitir toxocariasis humana en lugares públicos ha sido demostrado en todo el mundo (Overgaauw 2008). En los parques públicos de la Ciudad de México se reportó alta contaminación debida a la densidad de perros, con niveles del $60 \%$ (Romero 2009), semejantes a la frecuencia del $62.5 \%$ encontrada en el primer estudio realizado en Mexicali (Tinoco 2007).

La frecuencia de Toxocara canis en parques en 2016 fue de $46.4 \%(26 / 56)$ con un $11.7 \%(66 / 560)$ de muestras positivas, lo que indica una baja contaminación del suelo, ligeramente menor a la reportada en los jardines de la Universidad Metropolitana de México, que fue del 12.9\% (Trejo 2012). Cabe destacar que, aunque la frecuencia es baja, el riesgo de la parasitosis existe.

Durante el transcurso del año se encontró una importante variación estacional en la tasa de contaminación por Toxocara canis, lo que indica que el riesgo varía dependiendo de la temporada. El parásito puede encontrarse en cualquier estación del año, siendo 3.7 veces mayor el riesgo en primavera.

El clima de Mexicali es árido, muy seco y con variabilidad térmica extrema. Las temperaturas varían de 22 a $37^{\circ} \mathrm{C}$ de febrero a mayo, lo cual puede modificarse por la presencia de frentes fríos que llegan acompañados de viento, provocando descenso en la temperatura (García-Cueto 2007).

Estudios realizados en países con climas diferentes registraron variación estacional. En Hannover, Alemania, los periodos de mayor contaminación fueron los meses de enero a junio. Febrero fue el mes con la tasa más alta de contaminación por huevos infecciosos (41.3\%) (Kleine 2017). 
Salinas (2001) encontró que en las plazas de Santiago de Chile el riesgo era mayor durante el otoño $(29.5 \%)$, seguido de la primavera $(23.8 \%)$. Pezeshki (2017) encontró encontró una frecuencia global de $17.5 \%$ en Ardabil, Irán, correspondiendo $12 \%$ a Toxocara canis durante la primavera.

Sin embargo, diversos factores físicos, ambientales y metodológicos inciden en la recuperación de huevos de Toxocara en muestras de suelo. Esto dificulta la evaluación de variaciones estacionales en la contaminación del suelo por los huevos de parásitos y explica la escasez de este tipo de investigación (Laird 2000).

La supervivencia y el desarrollo de los huevos de Toxocara dependen de la temperatura, la humedad y la exposición al sol (Gamboa 2005). El desarrollo de los huevos comienza alrededor de los $4{ }^{\circ} \mathrm{C}$ y se acelera conforme aumenta la temperatura, pero los huevos se deshidratan a los $37^{\circ} \mathrm{C}$. De acuerdo con los resultados obtenidos, la mayor frecuencia de huevos de Toxocara canis en el suelo se encontró en áreas con pasto, dado que éstos pueden resguardarse del sol y conservar la humedad (Laird 2000).

La frecuencia de contaminación del parásito en el estudio de 2007 fue de $62.5 \%$ (20/32) mientras que en el de 2016 disminuyó a 46.4 \% (26/56), con una diferencia de $16.1 \%$.

A pesar de que las temperaturas superficiales de suelo en Mexicali llegan a superar los $37^{\circ} \mathrm{C}$, en los parques, que constituyen un microclima controlado debido al riego y la presencia de árboles, las temperaturas varían de 22 a $37^{\circ} \mathrm{C}$, lo que permite una mayor sobrevivencia de los huevos.

Cabe destacar que durante los meses de febrero a mayo se presentan frentes fríos que originan lluvias y decrementos en la temperatura de la ciudad. Diversos estudios han reportado disminuciones en las frecuencias de los parásitos, lo cual se atribuye a su ciclo biológico con la estacionalidad y el tipo de suelo (Nijsse 2016), así como al acceso de los perros a los parques (Saraei 2012).

La presencia de huevos de Toxocara canis se asocia con pobreza e insalubridad. Una de las posibles causas de la ligera disminución de la frecuencia en este estudio es que el muestreo fue realizado en parques públicos que son atendidos por la dirección municipal de áreas verdes de Mexicali. Estos parques se limpian y riegan tres veces por semana; no obstante, carecen de un método de disposición de heces adecuado, por lo que algunas veces se eliminan con la basura y otras son confinadas en algún rincón del parque. Sin embargo, se observó durante los muestreos que la gente lleva a sus mascotas a defecar a los parques y los propietarios no recogen las heces, por lo que el problema no se ha resuelto en su totalidad. Asimismo, la gran cantidad de perros callejeros que portan parásitos y los propagan sin ningún control en los suelos contribuyen a esta situación (Trasviña 2017).

Aparte de los huevos de Toxocara canis se observó la presencia de Ancylostoma caninum. El tercer estadio de la larva responde a las vibraciones del suelo y a las altas temperaturas, con movimientos parecidos a los de la culebra, lo que parece ayudarle a encontrar al huésped (Heukelbach 2008). Al penetrar en la piel también provoca la enfermedad de larva migrans cutánea (Bowman 2010). La frecuencia de A. canium en Mexicali es baja respecto de estudios realizados en otras partes del país como San Cristóbal de las Casas, Chiapas, donde se reportó una frecuencia de $18.5 \%$ (Martínez 2008). En Culiacán, Sinaloa, fue $25.3 \%$ (Rubio 2012).

Por otra parte, también se encontraron Strongylus spp. y Spirocerca spp. Considerando la baja frecuencia de estos parásitos reportada, debe contemplarse el impacto zoonótico en humanos y el riesgo parasitario en animales domésticos, respectivamente.

La densidad de contaminación por huevos de Toxocara canis en este estudio fue de 1.6 huevos por $66 \mathrm{~g}$ de suelo. A pesar del alto número de parques públicos contaminados $(30 / 52)$, el grado de contaminación de cada parque es bajo: 25 de los 30 parques parasitados resultaron tener de una a tres muestras positivas donde se encontraron también huevos infértiles. Esto puede atribuirse a las características del suelo y la disminución de cachorros en los parques, ya que excretan más huevos que los perros adultos (Archelli 2008) y posiblemente no sean llevados al parque hasta que son mayores (Jeyathilakan 2014).

En Mexicali, la disminución de Toxocara canis puede ser consecuencia de las campañas sanitarias y educativas por parte de los médicos veterinarios y asociaciones civiles contra la riquetsiosis. Se ha desparasitado a los perros con ivermectina, la cual también tiene actividad contra Toxocara canis (Ibarra 2015). Sin embargo, no se ha realizado una encuesta representativa de la población para comprobar esta hipótesis.

\section{CONCLUSIONES}

Los parques brindan beneficios sociales y ambientales, pero si no son cuidados por el gobierno y sobre todo por la comunidad, pueden convertirse en un problema de salud pública. 
Durante el año de muestreo en diferentes colonias de Mexicali, se observó que uno de cada dos parques está contaminado con al menos un parásito intestinal.

La frecuencia de parques con huevos de Toxocara canis disminuyó en $16.1 \%$ respecto al estudio realizado en 2007; sin embargo, la población humana y canina sigue estando en riesgo, lo cual se suma a la contaminación visual que causan las heces en la vía pública, ya que en todos los parques se detectó su presencia.

El fecalismo canino en la vía pública tiene su raíz en la tenencia irresponsable de mascotas, lo cual permite que los perros vayan al parque o deambulen por las calles liberando al dueño de la responsabilidad de la disposición final de las heces.

Las parasitosis son padecimientos que pueden disminuir conforme se apliquen las medidas de higiene adecuadas. Para ello las personas deben modificar creencias y conductas relativas a la forma en que ven a sus mascotas.

Se sugiere realizar estudios serológicos para determinar la exposición humana al parásito, implementar biodigestores de heces en los parques para una su mejor disposición, continuar con las campañas de sensibilización reforzando el concepto de una sola salud, en el entendido de que la calidad de vida humana está directamente ligada a la calidad de vida animal.

\section{AGRADECIMIENTOS}

Esta investigación se realizó con fondos del Consejo Nacional de Ciencia y Tecnología de México (CONACyT). Se agradece el apoyo y dirección durante todo el proceso de los doctores Margarito Quintero, Sergio Cueto, Luis Tinoco y Enrique Trasviña del Instituto de Investigaciones en Ciencias Veterinarias de la Universidad Autónoma de Baja California. Asimismo, agradecemos a los doctores Rafael García, Socorro Romero y Jaime Reyes del Instituto de Ingeniería de la Universidad Autónoma de Baja California, por las facilidades y atenciones para la realización de este proyecto.

\section{REFERENCIAS}

Alvarado-Esquivel C. (2014). Seroepidemiology of toxocariasis in a rural Tepehuanos population from Durango, Mexico. J. Helminthol. 88 (2), 173-6. DOI: $10.1017 / \mathrm{S} 0022149 \mathrm{X} 12000880$

Archelli S. (2008). Toxocara y toxocariosis. Acta Bioquím. Clín. L. 42 (3), 379-384.
Avcioglu H, B. A. (2008). Seasonal prevalence of Toxocara ova in soil samples from public parks in Ankara, Turkey. Vector Borne Zoonotic Dis, 345-350.

DOI: $10.1089 / v b z .2007 .0212$

Bowman D. (2010). Hookworms of dogs and cats as agents of cutaneous larva migrans. Trends Parasitol. 26 (4), 62-67. DOI: 10.1016/j.pt.2010.01.005

Carvalho E. (2011). Toxocariasis: Visceral larva migrans in children. J. Pediatr. (Rio J.). 87 (2), 100-10. DOI: 10.1590/S0021-75572011000200004

CDC (2017). Toxocariasis FAQs. Centers for Disease Control and Prevention [en línea]. https://www.cdc.gov/ parasites/toxocariasis/gen_info/faqs.html 7/04/2017

Gamboa M. (2005). Effects of temperature and humidity on the development of eggs of Toxocara canis under laboratory conditions. J. Helminthol. 79 (4), 327-331. DOI: $10.1079 / \mathrm{JOH} 2005287$

GIDEON (2017). Outbreak and diseases. Global Infectious Disease and Epidemiology Network [en línea]. https:// web.gideononline.com/web/epidemiology/index.php? country $=$ OUTBREAK $\&$ disease $=12370 \&$ view $=$ Distri bution\# 8/09/2017

Gobierno del Estado de Baja California (2013). Diagnóstico estratégico. Gobierno de Baja California, México [en línea]. http://www.bajacalifornia.gob.mx/ portal/gobierno/ped/doctos/diagnostico_estrategico. pdf $7 / 09 / 2017$

Heukelbach J. (2008). Epidemiological and clinical characteristics of hookworms-related cutaneous larva migrans. Lancet Infect. Dis. 8 (5), 302-309. DOI: 10.1016/S1473-3099(08)70098-7

Holland C. (2005). Toxocara: The enigmatic parasite. CABI Publising, Dublin, Irlanda, $301 \mathrm{pp}$.

Ibarra F. (2015). Comparison of two pour on formulations of ivermectin against gastrointestinal worms, fleas and lice in naturally infected stray dogs. Pharmacology and Pharmacy 6 (3), 177-184.

DOI: $10.4236 /$ pp.2015.63018

IMIP (2016). Mapa de tipología de vivienda en Mexicali. Instituto Municipal de Investigación y Planeación Urbana de Mexicali, Mexicali, B.C., México, 1 pp.

ISUCFSPH (2016). Toxocariasis. Iowa State University Center for Food Security and Public Health [en línea]. https://lib.dr.iastate.edu/cfsph_factsheets/134 9/09/2017

Jeyathilakan D. (2014). Detection of Toxocara eggs in contaminated soil from various public places of Chennai city and detailed correlation with literature. J. Parasit. Dis. 38 (2), 174-180.

DOI: $10.1007 / \mathrm{s} 12639-012-0217-\mathrm{x}$

Katagiri S. (2008). Prevalence of dog intestinal parasites and risk perception of zoonotic infection by dog owners in Sao Paulo State, Brazil. Zoonoses Public Hlth. 55 (8), 406-413. DOI: 10.1111/j.1863-2378.2008. 01163.x 
Kazacos K. (2000). Protecting children from helminthic zoonoses. Contemp. Pediatr. 95 (3), 1-24.

Kleine A. (2017). Seasonal variation in the prevalence of Toxocara eggs on children's playgrounds in the city of Hanover, Germany. Parasite. Vector. 10 (248).

DOI: 10.1186/s13071-017-2193-6

Laird R. (2000). Toxocara spp. en parques y zonas públicas de la Ciudad de la Habana. Rev. Cubana Hig. Epidemiol. 38 (2), 112-116.

Martínez I. (2008). Contaminación parasitaria en heces de perros, recolectadas en calles de ciudad de San Cristóbal de Las Casas, Chiapas, México. Vet. México 39 (2), 173-180.

Nava N. (2015). Presence of anti Toxocara canis antibodies and risk factors in children from the Amecameca and Chalco regions of México. BMC Pediatrics 15 (65). DOI: $10.1186 / \mathrm{s} 12887-015-0385-9$

Nijsse R. (2016). Recurrent patent infections with Toxocara canis in household dogs older than six months: A prospective study. Parasite. Vector. 9 (1), 531. DOI: 10.1186/s13071-016-1816-7

Oliveira A. (2017). Molecular identification of Ancylostoma species from dogs and an assessment of zoonotic risk in low-income households, Sao Paulo State, Brazil. J. Helminthol. 91 (1), 14-19. DOI: $10.1017 / \mathrm{S} 0022149 \mathrm{X} 15001145$

OMS (2017). Helmintiasis transmitidas por el suelo. Organización Mundial de la Salud [en línea]. http://www. who.int/mediacentre/factsheets/fs366/es/ 7/04 2017

Overgaauw P. (2008). Aspects of Toxocara epidemiology: Human toxocarosis. Crit. Rev. Microbiol. 23 (3), 215231. DOI: $10.3109 / 10408419709115137$

Pezeshki A. (2017). Detection of Toxocara spp. eggs in the soil of public places in and around of Ardabil City, Northwestern Iran. Iran J. Parasitol. 12 (1), 136-142.

Romero C. (2009). Contaminación por Toxocara spp. en parques de Tulyehualco, México. Rev. Cient. Fac. Cien. V. 19 (3), 253-256.
Romero C. (2013). Prevalence and risk factors associated with Toxocara canis infection in children. Sci. World J. 2013, 572089. DOI: 10.1155/2013/572089

Rubio C. (2012). Canine parasites in soil of public recreation areas in Culiacan, Sinaloa, Mexico. Int. J. Infect. Dist. 16, e461. DOI: 10.1016/j.ijid.2012.05.665.

Ruiz de Ybáñez R. (2001). Prevalence and viability of eggs of Toxocara spp. and Toxocara leonina in public parks in Eastern Spain. J. Helminthol. 75 (2), 169-173. DOI: $10.1079 / \mathrm{JOH} 200164$

Salinas P. (2001). Prevalencia de hallazgo de huevos de Toxocara canis en plazas de la región metropolitana de la ciudad de Santiago, Chile. Boletín Chileno de Parasitología 56 (3), 102-105.

DOI: $10.4067 / \mathrm{S} 0365-94022001000200013$

Saraei M. (2012). Contamination of soil and grass to Toxocara spp. eggs in public parks of Qazvin, Iran. Asian Pac. J. Trop. Biomed. 2 (1), S1156-S1158. DOI: 10.1016/S2221-1691(12)60377-3

Tinoco L. (2007). Frequency of Toxocara canis eggs in public parks of the urban area of Mexicali, B.C. Mexico. J. Anim. Vet. Adv. 6 (3), 430-434.

Tinoco L. (2008). Seroprevalence of larva migrans of Toxocara canis and evaluation of associated risk factors among children in a Mexico-United States border region. Intern. J. Appl. Res. Vet. Med. 6 (2), 130-136.

Trasviña E. (2017). Prevalence and distribution of intestinal parasites in stray dogs. Austral. J. Vet. 49 (2), 105-111. DOI: 10.4067/S0719-81322017000200105

Trejo C. (2012). Soil contamination by Toxocara spp. eggs in a university in Mexico City. Rev. Bras. Parasitol. Vet. 21 (3), 298-300. DOI: $10.1590 / \mathrm{S} 1984-29612012000300022$

Zajac A. (2012). Veterinary clinical parasitology. John Wiley and Sons, Oxford, Reino Unido, 354 pp. 